\title{
Costilla o columna, la mujer como proveedora de servicios sociales
}

Marga Marí-Klose, Anna Nos Colom y Pau Marí-Klose*

\section{El Estado del malestar}

La crítica desde la perspectiva de género a las distintas caracterizaciones de los modelos de Estado del Bienestar subraya la insuficiencia explicativa de estas aproximaciones al olvidar la categoría de género. Las teorías feministas consideran que el análisis de los Estados de Bienestar precisan la ampliación del modelo explicativo clásico basado en la relación Estado-mercado introduciendo un tercer ingrediente: la familia. Todos los regímenes de bienestar social modernos han operado a partir de alguna idea previa en materia de política familiar, si bien en po- cas ocasiones se ha explicitado. A partir del momento en que comienza a prestarse una seria atención al papel de la familia se hace necesario conceptualizarla también como proveedora y no sólo como receptora de prestaciones sociales y considerarla en términos de su relación con el mercado de trabajo, las transferencias y los servicios. En el momento en que la familia es considerada como proveedora de bienestar -como un elemento primordial de protección social- aparece en primer plano el debate sobre la división entre trabajo remunerado y no remunerado '. Es en el ámbito doméstico donde la mujer realiza buena parte de su actividad productora y reproductora. El problema surge cuando la actividad doméstica, tradicionalmente asignada a las mujeres, no se ha definido como trabajo. El concepto de "trabajo" se consideraba sinónimo de empleo, es decir, entendido como aquella actividad que vincula trabajo y remuneración excluyendo toda aquella actividad no asalariada ${ }^{2}$. En consecuencia, el discurso sobre el que se articulan las teorías clásicas del Estado del Bienestar ha permanecido "ciego al sexo" ${ }^{3}$.

Como señala Duncan (1995), las mujeres desaparecen del análisis en el momento en que no participan $\multimap$ al menos no en las mismas condiciones que los hombres- en el mercado laboral, ya que en los esquemas desarrollados el trabajo doméstico y las tareas de cuidado no merecen ninguna importancia. Esta desatención a la actividad femenina repercute sobre la consideración de las mujeres (no asalariadas) como ciudadanas de derecho ${ }^{4}$. Los derechos de participación ciudadana tienen una dimensión de género. Muchos de los derechos de la ciudadanía social, en buena parte, siguen consistiendo en garantías sociales que hacen referencia al ciudadano «varón, en tanto que empleado asalariado, en lugar del conjunto de la ciudadanía —varones y mujeres- En consecuencia, contingencias típicas de las formas de vida masculinas (como jubilaciones, seguro de accidentes, desocupación) están, en todos los países, más incorporadas en los programas sociales que los de las mujeres (viudedad, cuidado familiar y matemidad). Los sistemas de bienestar han tendido a reforzar la tradicional división del trabajo y la dependencia entre hombres y mujeres (GORDON, 1990).

Las políticas de bienestar tienden a reproducir las estructuras patriarcales de la sociedad. Desde una perspectiva marxista, Heidi HaRTMANN señala que la emergencia del Estado de Bienestar en el sistema capitalista sería una transición de un patriarcado familiar a un patriarcado estructural. La esfera de la reproducción (tradicionalmente femenina) se subordina a la de la producción (masculina). La incorporación de las mujeres a la vida pública no ha supuesto el abandono de las responsabilidades que se corresponden a las obligaciones domésticas: la reproducción continúa siendo un espacio invisible y sin reconocimiento social.

La familia parece que camina hacia unos modelos en los que los roles son intercambiables, pero todavía para la mayoría de las mujeres casadas, incluidas muchas de aquellas que tienen un trabajo remunerado, se considera que su rol principal es 
el de amas de casa y madres ${ }^{5}$. El éxodo de las mujeres al mercado no viene acompañado por un cambio cultural en el modelo de familia y trabajo que facilite esa transición. Cambia la fuerza de trabajo; cambian las mujeres. Sin embargo, las características de los puestos de trabajo permanecen inflexibles e impermeables a esos cambios. La tensión que se deriva de la inadaptación del mercado a las exigencias de compatibilidad del trabajo remunerado y doméstico llevan a hablar a Arlie HoCHSHIELD sobre la stalled revolution. El varón no se ha incorporado a la esfera doméstica, el mercado laboral no se ha adaptado a las necesidades de las mujeres y el Estado de "Bienestar" no ha ręmediado el malestar que esta nueva situación produce en lä vida de las mujeres.

Lo que se plantea desde una perspectiva feminista es poner de relieve el hecho de que los modos como se construye un Estado del Bienestar y las formas de interacción entre la política social y el mercado de trabajo pueden influir en la reproducción de roles familiares y las posibilidades de producción de nuevos roles, al suponer bien sea incentivos o desincentivos a la búsqueda de empleo de las mujeres fuera del hogar y a la permanencia en el mercado de trabajo (Guillén, 1996).

\section{El desmantelamiento de la (des)protección social pública: un debate estéril}

En época de vacas flacas, todo el mundo vuelve su mirada hacia la familia. Las familias funcionan como agencias dedicadas a cuidar, alimentar y proporcionar cobijo a aquellos miembros más vulnerables frente a la acción del mercado: niños, ancianos, enfermos, parados y discapacitados. Sin embargo, la consolidación del Estado del Bienestar en Europa, y en particular la extensión de un cierto consenso socialdemócrata (en su sentido lato) en tomo a estas cuestiones, habían servido para que el Estado adquiriera responsabilidades directas en la atención a este tipo de personas, suplantando en muchos casos a la familia a través de servicios sociales personales. Esto permitió transformar en este campo la estructuración corporativista de algunos Estados del Bienestar, asentada sobre el "principio de subsidiariedad", según el cual el Estado sólo debe proveer estos servicios cuando se agota la capacidad de provisión de la familia. Ahora, en el fragor del debate en torno a la viabilidad financiera de las estructuras públicas de bienestar, se alzan las voces de quienes piden traspasar de nuevo a la familia todas las responsabilidades asistenciales. En la mayor parte de Europa esta propuesta entra en conflicto, por un lado, con la creciente ines- tabilidad familiar, y, por otro, con el deseo de las mujeres de desarrollar sus carreras profesionales (ESPING-ANDERSEN, 1996: 368).

En España, donde estas tendencias también empiezan a cobrar importancia, hay que tener en cuenta, además, que la familia ya sobrelleva una carga demasiado pesada, que está condicionando en gran medida los itinerarios de vida de muchas mujeres españolas. El sociólogo Julio IGLESIAS DE Ussel señala factores de tipo demográfico, social y político: ael espectacular incremento de la esperanza de vida de las últimas décadas, unido a la más reciente caída de la fecundidad, genera un notable desequilibrio entre los prestadores y destinatarios de servicios domésticos. En segundo lugar, la masiva incorporación de la mujer a la educación y al mercado de trabajo hace disminuir drásticamente el colectivo de personas tradicionalmente dispuestas a prestar este tipo de asistencia. Por último, la crisis del Estado del Bienestar y el giro radical experimentado por el discurso público en torno a estas materias colocan sobre las espaldas de la familia una mayor carga de la que cabría esperar ${ }^{6}$. Todo lo que signifique recortar prestaciones asistenciales y programas de apoyo a la familia, y no expansión y desarrollo, tiene un repercusión directa sobre la trayectoria vital de las mujeres cuya aportación al abastecimiento de atenciones y bienestar continúa siendo decisiva.

El Estado del Bienestar español ha experimentado en los últimos quince años una expansión sin precedentes. El resultado final es el afianzamiento de la asistencia sanitaria pública y del sistema de pensiones de jubilación, que proporcionan cobertura a todos los españoles. Sin embargo, otros sistemas de protección como los servicios sociales personales o algunos programas asistenciales, aun habiendo mejorado considerablemente, permanecen, en comparación con los Estados del Bienestar europeos más avanzados, en un estado de subdesarrollo. Esto no ha supuesto un déficit significativo en el abastecimiento de bienestar y cuidados gracias al papel crucial de la familia, y en particular al sacrificio colectivo que llevan a cabo las mujeres en el seno de la familia ${ }^{7}$. El Estado del Bienestar español se caracteriza por pedir grandes esfuerzos a las familias y ofrecerles pocas ayudas a cambio (Gullés, 1996). En alguno de los capítulos más importantes en que se centra este esfuerzo, donde se dice familia tendría que hablarse simplemente de mujeres: en especial, en todo lo que se refiere a cuidados de niños/as, ancianos/as, enfermos/as y discapacitados/as.

La. gran mayoría de las familias no pueden enviar a sus hijos a guarderías públicas. En España la oferta pública de plazas para niños/as de dos años y menos es prácticamente inexistente (1\%), situándose por debajo incluso de los niveles de los países europeos más atrasados en este apartado (Grecia 4\% y Portugal 6\%) (VALIENTE, 1995). Tampoco se han desarrollado servicios prestados a los niños en horario extra-escolar. Esta precariedad del sistema de servicios sociales dedicado a cuidar y atender a 
niños de corta edad no viene compensada por una adecuada patibilidad del rol profesional y el rol familiar de las mujeres tal política de prestaciones y subsidios a fin de garantizar la com- y como se demuestra en la Tabla 1.

\begin{tabular}{|c|c|c|c|c|}
\hline \multicolumn{5}{|l|}{ 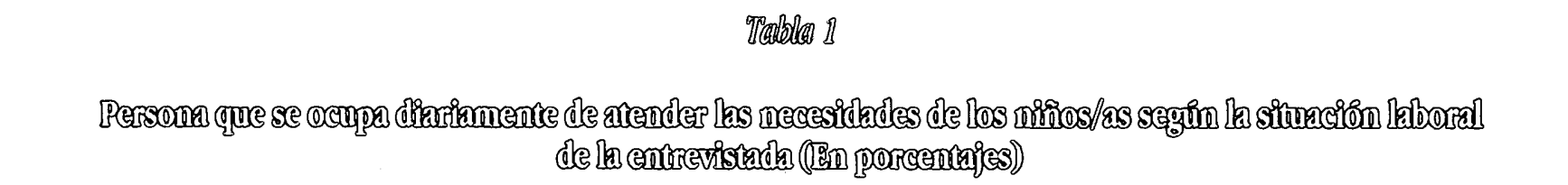 } \\
\hline \multirow{2}{*}{ Persona que cuida diariamente del niño/a } & \multicolumn{4}{|c|}{ Situación laboral de la entrevistada } \\
\hline & Total & Trabaja & Trabajó anteriormente & Nunca ha trabajado \\
\hline La madre & $84 \%$ & $65 \%$ & $96 \%$ & $94 \%$ \\
\hline El padre & 1,9 & 4,3 & 0,4 & 0,3 \\
\hline Ambos & 9,3 & 19 & 3,1 & 4,2 \\
\hline Otro familiar & 5,6 & 14 & 0,6 & 1,0 \\
\hline Enipleada de hogar & 3,7 & 9,6 & - & - \\
\hline $\mathrm{Ns} / \mathrm{nc}$ & 0,3 & 0,4 & 0,1 & 0,3 \\
\hline Total & $100 \%$ & & & \\
\hline Número de casos & 2.000 & 761 & 928 & 311 \\
\hline
\end{tabular}

Fuente: Expectativas sobre redes de cuidados de bijos.

(Madrid: Instituto de la Mujer, 1990), p. 11

En la gran mayoría de los casos la madre es la persona que se ocupa diariamente, y en exclusiva de atender las necesidades de sus hijos/as. Sólo una de cada diez mujeres comparte estas tareas a partes iguales con su compañero, mientras que en otro $10 \%$ de los casos corre a cargo de otros familiares o personas contratadas al respecto. La situación laboral de la mujer también determina algunas diferencias de comportamiento: la solución compartida o ajena al núcleo de la madre y el padre (otros familiares o em- pleadas) supone hasta un $40 \%$ de casos entre las mujeres que trabajan, frente a un $5 \%$ entre las que no lo hacen. A todo esto cabe añadir que existe un estigma sobre las madres que no atienden personalmente las "necesidades" de sus hijos/as: «existe un fuerte control social y profesional (médicos y psicólogos) que defiende la idea de que los bebés y los niños pequeños deben pasar el mayor tiempo posible con sus madres, idea reforzada por la mentalidad tradicional. (GulLén, 1997:57) ${ }^{8}$.

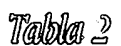

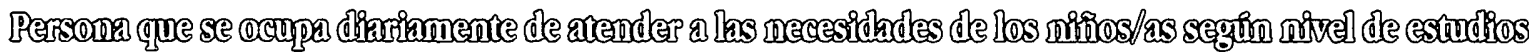

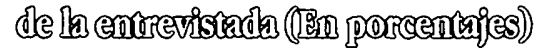

\begin{tabular}{|c|c|c|c|c|c|c|c|c|}
\hline $\begin{array}{l}\text { Persona que cuida diariamente } \\
\text { del niño/a: }\end{array}$ & \multicolumn{8}{|c|}{ Nivel de estudios de la entrevistada } \\
\hline La madre & $84 \%$ & & $95 \%$ & $91 \%$ & $88 \%$ & $77 \%$ & $64 \%$ & $62 \%$ \\
\hline El padre & 1,9 & $\cdot$ & 1,0 & 0,8 & 1,0 & 2,9 & 6,8 & 3,6 \\
\hline Ambos & 9,3 & & 1,0 & 3,8 & 6,6 & 15 & 23 & 28 \\
\hline Empleada de hogar & 3,7 & & - & 0,8 & 3,1 & 4,0 & 12 & 12 \\
\hline $\mathrm{Ns} / \mathrm{nc}$ & 0,3 & & 0,5 & 0,7 & - & - & - & - \\
\hline Total & $100 \%$ & & & & & & & \\
\hline Número de casos & 2.000 & & 198 & 600 & 604 & 276 & 176 & 138 \\
\hline
\end{tabular}

Fuente: Expectativas sobre redes de cuidados de bijos (Madrid: Instituto de la Mujer, 1990), p. 118. 
A medida que aumenta el nivel de estudios se eleva también la proporción de familias donde esa labor diaria de atención al niño/a es compartida por el padre y la madre (Tabla 2).

El cambio en las relaciones padres-hijos ha sido, junto a la incorporación de la mujer al mercado laboral, el principal factor de modificación de la familia. Se puede afirmar que para el caso español no son las transformaciones familiares las que favorecen o permiten el cambio en el rol femenino (como se observa grosso modo en Europa donde además las políticas de bienestar se encuentran más desarrolladas) sino que es el nuevo papel económico de la mujer lo que va a impulsar a la institución familiar hacia nuevas formas. Según la Tabla 3, entre las mujeres que trabajan se rechaza mayoritariamente que el trabajo de la mujer interfiera negativamente en la relación con sus hijos. A su vez, entre las que trabajan, a medida que aumenta el número de hijos disminuye el porcentaje de mujeres que se muestran de acuerdo con la frase: una madre que trabaja puede tener con sus hijos una relación tan cálida y estable como una madre que no trabaja.. En cambio, las mujeres que se dedican a las labores domésticas se muestran mayoritariamente en desacuerdo. A medida que aumenta el número de hijos se incrementa la tendencia a pensar que el trabajo interfiere en las relaciones con los hijos/as. La mayoría de las mujeres que trabajan (51\%) consideran que un niño en edad preescolar no se ve perjudicado porque su madre trabaje. Esta opinión cambia si las mujeres tienen tres o más hijos. Se trata de mujeres con importantes cargas familiares que encuentran dificultades a la hora de compatibilizar las dos esferas (doméstica y laboral).

A diferencia de los varones, cuya identidad cultural se sustenta a partir de su ocupación en el espacio público y en concreto en el ejercicio de un trabajo remunerado, a las mujeres no se les atribuye una cultura del trabajo. Según la Tabla 3, la realización de la mujer como madre y esposa encuentra resistencias entre las mujeres que trabajan con menos de tres hijos. Las normas de conducta de género han influido de forma significativa en la consideración del trabajo extradoméstico femenino. Como afirma Mary NASH, «en la medida en que se reconoce su realidad es considerado como una desvirtuación de su sublime 'misión' de madre y. "ángel del hogar" ${ }^{\prime \prime}{ }^{9}$. Las mujeres que se dedican a las labores domésticas se muestran mayoritariamente de acuerdo en consonancia con la frase atrabajar está bien, pero lo que las mujeres realmente quieren es crear un hogar de las mujeres sin hijos, que se muestran en desacuerdo, bien porque no han completado su proceso de domesticación (aún no son madres), o bien porque son jóvenes (y por tanto existe un cambio de mentalidades).

Muchas prestaciones que tienen un carácter universal en otros países, sólo son residuales en España (MADRuga, 1996: 390). El nivel salarial máximo para disfrutar de estos servicios es muy bajo: un millón de pesetas, cantidad sólo ligeramente su- perior al salario mínimo interprofesional (en RodRíguez CABRERO, 1994, citado en Gullén, 1997: 51). Las comparaciones con otros países de la Unión Europea mueven al sonrojo. En España los subsidios familiares por joven menor de veinte años a cargo representan el 0,5\% del PIB, a distancia astronómica (treinta veces menor) del país que más gastaba, Dinamarca. En términos del porcentaje sobre el salario medio neto, los subsidios familiares en España suponen el 2\%, porcentaje sólo superior al de Francia (GuilLén, 1997:51).

Los déficit del Estado del bienestar a las demandas surgidas en el seno de la familia se resuelven en el propio núcleo familiar a través de división sexual del trabajo. Como señala Jesús M. de Miguel, ala familia española es gradualmente menos escuela, menos hospital y menos seguridad socialn. Pero todavía . conserva numerosas funciones de agente cuidador y de ayuda psicológica y económica a sus miembros/as. [... LLas redes de solidaridad familiar en España siguen siendo importantes. Los jóvenes no abandonan la casa de sus padres, y hay bastantes abuelos que viven en casa de sus hijos/as. La importancia de las relaciones familiares en España se puede observar con otros indicadores. La tasa de camas hospitalarias es la mitad que la media europea, porque las camas thospitalarias están dentro de los hogares. Las mujeres 'amas de casa' cuidan a enfermos/as crónicos, familiares discapacitados, minusválidos, parados/as e hijos/as no integrados" ${ }^{10}$. Con la maternidad se inicia la responsabilidad de la mujer en los cuidados de la familia. Los niños/as que no asisten a centros escolares o guarderías pasan el tiempo mayoritariamente en sus casas y con sus madres. En un estudio realizado por el Instituto de la Mujer en 1990, según muestran los datos en el $82 \%$ de los casos son las madres la que se ocupan del cuidado de sus hijos/as cuando no asisten a guarderías o centros escolares. El papel desempeñado por el padre, en este sentido, es prácticamente residual, siendo más importante el de los abuelos/as y las personas contratadas, especialmente cuando las madres trabajan fuera del hogar. En estos casos, como señala el mismo estudio, las mujeres con horarios más extensivos o con unas condiciones laborales menos flexibles concentran las situaciones más problemáticas en la compatibilidad maternidad-empleo. La actual situación de la mujer plantea la disyuntiva esquizoide de poder optar al éxito tanto mediante la carrera familiar como mediante la carrera ocupacional: esto genera no sólo la sobrecarga de la doble jornada sino además el encierro en un dilema de indecisión racional semejante al ejemplo escolástico del asno de Buridán. Se trata de la figura topológica conocida como bifurcación, por abrirse una encrucijada de alternativas equiprobables (las dos carreras, ocupacional y familiar) entre las que no se puede elegir más que irracionalmente (por amor, al azar o supersticiosamente) ${ }^{11}$.

El capítulo de los derechos que protegen la maternidad de las mujeres que trabajan está más desarrollado (baja de materni- 
Texide 3)

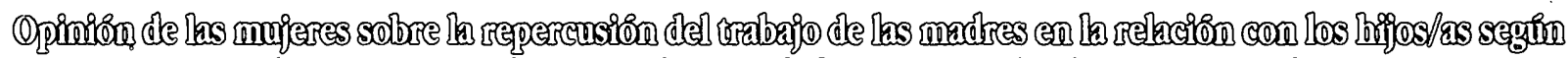

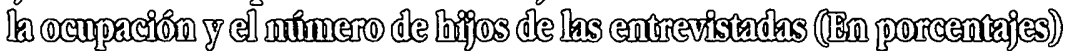

\begin{tabular}{|c|c|c|c|c|c|c|c|c|}
\hline \multirow{3}{*}{$\begin{array}{l}\text { Una madre que trabaja puede } \\
\text { tener con sus hijos una } \\
\text { relación tan cálida y estable } \\
\text { como una madre que no } \\
\text { trabaja }\end{array}$} & \multicolumn{8}{|c|}{ Ocupación } \\
\hline & \multicolumn{4}{|c|}{ Trabaja } & \multicolumn{4}{|c|}{ Labores domésticas } \\
\hline & Total & Sin hijos/as & $\begin{array}{l}\text { Uno o dos } \\
\text { hijos/as }\end{array}$ & Tres o más & Total & Sin hijos/as & $\begin{array}{l}\text { Uno o dos } \\
\text { hijos/as }\end{array}$ & Tres o más \\
\hline Indiferente & 2,3 & 2,4 & 1,9 & 3,2 & 3,9 & 6,1 & 3,8 & 3,7 \\
\hline En desacuerdo & 28 & 21 & 29 & 40 & 49 & 33 & 48 & 54 \\
\hline Total & $100 \%$ & & & & & & $(234)$ & (215) \\
\hline Número de casos & (348) & (125) & (348) & (63) & (482) & (33) & & \\
\hline
\end{tabular}

\begin{tabular}{|c|c|c|c|c|c|c|c|c|}
\hline \multirow{4}{*}{$\begin{array}{l}\text { A un niño/a en edad } \\
\text { preescolar le puede } \\
\text { perjudicar que su } \\
\text { madre trabaje }\end{array}$} & \multicolumn{8}{|c|}{ Ocupación } \\
\hline & \multicolumn{4}{|c|}{ Trabaja } & \multicolumn{4}{|c|}{ Labores domésticas } \\
\hline & \multicolumn{4}{|c|}{ Número de hijos/as } & \multicolumn{4}{|c|}{ Número de hijos/as } \\
\hline & Total & Sin hijos/as & $\begin{array}{l}\text { Uno o dos } \\
\text { hijos/as }\end{array}$ & Tres o más & Total & Sin hijos/as & $\begin{array}{l}\text { Uno o dos } \\
\text { hijos/as }\end{array}$ & Tres o más \\
\hline De acuerdo & $38 \%$ & $34 \%$ & $38 \%$ & $49 \%$ & $61 \%$ & $53 \%$ & $58 \%$ & $66 \%$ \\
\hline Indiferente & 11 & 8,2 & 13 & 9,2 & 12 & 16 & 14 & 8,2 \\
\hline En desacuerdo & 51 & 57 & 50 & 41 & 27 & 31 & 28 & 26 \\
\hline Total & $100 \%$ & & & & & & & \\
\hline Número de casos & (234) & (122) & (157) & (61) & (122) & (32) & (229) & $(207)$ \\
\hline \multirow{4}{*}{$\begin{array}{l}\text { Trabajar está bien, pero } \\
\text { lo que las mujeres } \\
\text { realmente qujeren es } \\
\text { crear un hogar }\end{array}$} & \multicolumn{8}{|c|}{ Ocupación } \\
\hline & \multicolumn{4}{|c|}{ Trabaja } & \multicolumn{4}{|c|}{ Labores domésticas } \\
\hline & \multicolumn{4}{|c|}{ Número de hijos/as } & \multicolumn{4}{|c|}{ Número de hijos/as } \\
\hline & Total & Sin hijos/as & $\begin{array}{l}\text { Uno o dos } \\
\text { hijos/as }\end{array}$ & Tres o más & Total & Sin hilos/as & $\begin{array}{l}\text { Uno o dos } \\
\text { hijos/as }\end{array}$ & Tres o más \\
\hline De acuerdo & $34 \%$ & $30 \%$ & $31 \%$ & $49 \%$ & $59 \%$ & $419 \%$ & $58 \%$ & $62 \%$ \\
\hline Indiferente & 11 & 10 & 12 & 7,9 & 12 & 12 & 11 & 13 \\
\hline En desacuerdo & 56 & 60 & 57 & 43 & 29 & 47 & 30 & 25 \\
\hline Total & $100 \%$ & & & & & & & \\
\hline Número de casos & (339) & (124) & (152) & (63) & (468) & (32) & (230) & (206) \\
\hline
\end{tabular}

Fuente: Datos del Banco de datos del Centro de Investigaciones Sociológicas CIS. Muestra nacional de 2.494 entrevistas, 1.292 mujeres de 18 y más años.

Estudio 2.113, septiembre 1994.

dad, parental, flexibilidad en la jomada laboral para compatibilizar el rol de madre y de trabajadora), aunque no consigue, por sí solo, los objetivos que se marca ${ }^{12}$. En estas condiciones las estructuras públicas de protección social en España han contribuido escasamente a transformar los esquemas a los que la mujer, en cuanto madre, tradicionalmente ha estado sometida ${ }^{13}$. Esto contribuye a explicar por qué las trayectorias vitales de las mujeres siguen asemejándose en muchos casos a las de sus madres y abuelas desde el momento en que se casan y comienzan a tener hijos. Los casos que se desvían de esta pauta, ciertamente cada vez más numerosos, se producen gracias a que algunas mujeres, merced a sus crecientes logros educativos y la independencia económica que les proporcionan sus trabajos remunerados, han podido ir a buscar al mercado lo que las es- 
tructuras públicas de protección social no les ofrecían, o bien han podido descargar las responsabilidades que culturalmente les son asignadas en cuanto madres sobre los hombros de otros miembros de la familia, casi siempre también mujeres ${ }^{14}$.

La sociedad de finales del siglo XX está haciendo frente a un fenómeno sin precedentes: el incremento de la longevidad. El importante cambio en la estructura de edad de la población está condicionado por el aumento de la esperanza de vida y del número de personas de edad avanzada; y el envejecimiento de la población propiciado además por la reducción de las tasas de natalidad ${ }^{15}$. Las mujeres, reflejando su mayor capacidad de supervivencia, representan el $60 \%$ de esta población. Casi el $70 \%$ de las personas de más de 80 años son mujeres. La combinación de la pauta de envejecimiento con la mayor supervivencia de la mujer dará lugar a una población mayor muy anciana y básicamente femenina. Dado que las mujeres se quedan viudas más frecuentemente que los hombres, tienden a vivir solas $o$, en menor medida, con sus hijos. Los hombres ancianos viven básicamente con sus esposas y cuando se quedan solos van a vivir con los hijos. El bajo porcentaje de ancianos en residencias muestra el rechazo tradicional hacia ese tipo de instituciones y el importante papel de la familia en el cuidado de ancianos que se quedan solos y necesitan ayuda. Parte de la demanda de los ancianos se dirige a los servicios públicos, tanto sanitarios como asistenciales. La oferta de servicios públicos para atender a necesidades no sanitarias de los ancianos (aseo, compra, gestiones, etc.) es una novedad relativa en los ayuntamientos españoles, que se ha visto rápidamente sobrepasada por el crecimiento de la demanda y las listas de espera. El sistema asistencial/sanitario público apenas ofrece cuidados totales a través de sus instituciones. El número de residencias está muy por debajo de la demanda actual, y más lejano todavía de la demanda potencial. Tampoco el sistema asistencial/sanitario privado actual es accesible, por su precio, a la mayoría de los ancianos. (M. Carmen Herrera y M. Ángeles DuRáN, 1995: 136)

Los sistemas de protección a los ancianos presentan bastantes deficiencias que repercuten directamente sobre las familias y quienes en el seno de éstas dedican horas a cuidar de las personas mayores, las mujeres. La oferta pública de plazas residenciales (12.500) es insignificante. No cubre ni siquiera el 0,5\% de la población anciana (Josep RodríguEz, 1994: 58). En muchas zonas de España, los ancianos/as no disponen todavía de la adecuada atención médica especializada y preventiva, ni centros de día próximos a su lugar de residencia. Las personas que recibieron atención domiciliaria son 25.000 . Esta cantidad minúscula parece sugerir que, sin conocer a ciencia cierta la demanda real de estos servicios, la oferta es insuficiente (Guiluén, 1997: 54). A falta de estos servicios, es frecuente que las ancianas se ocupen de los ancianos, que los ancianos vivan en casa de alguno de sus hijos/as casados/as y que la mujer adulta se encargue de cuidar de ellos, o que las hijas/nueras tengan que desplazarse con asiduidad al hogar de sus padres/suegros a velar por sus necesidades $\stackrel{16}{\circ}$

La familia, a pesar de las transformaciones y cambios que está experimentando, sigue considerándose a final del siglo XX como una de las instituciones firmemente establecidas y como proveedora de soporte-ayuda-atención a las personas ancianas en momentos de crisis decisiva. Éste es, precisamente, uno de los aspectos que confirma el reciente estudio sobre condiciones de vida y hábitos de la población (IEM, 1992); cuando las personas se hallan confrontadas a situaciones difíciles acuden a la familia; el caso de enfermedad es el más llamativo y que va en aumento (desde 1985) para las personas mayores de sesenta y cinco años de ambos sexos, aunque no sea ésa la única dificultad señalada (Carmen Domínguez, 1993). La red de solidaridad primaria, especialmente mediante los hijos/as, sale al paso frecuentemente de las necesidades de ayuda personal de los ancianos reduciendo su demanda de servicios sociales. No existe ningún tipo de retribuciones para estas familias (sería más propio decir para estas mujeres), lo que pone en evidencia hasta qué punto está arraigada la idea de que la asistencia a personas ancianas es fundamentalmente una obligación familiar (WARMAN, 1996). Las propuestas políticas elaboradas hacen referencia exclusivamente al anciano y no a aquellos que cuidan de él.

El cuidado familiar de los ancianos no ha entrado todavía en la dinámica política, ni como solución a las necesidades asistenciales de algunos ancianos ni como situación problemática para los cuidadores (o más bien cuidadoras). No se tiene en cuenta socialmente la labor asistencial de la familia y se menosprecia políticamente su contribución a la solución de problemas sociales. Las políticas que están siendo potenciadas son aquéllas dirigidas a fortalecer el papel de ayuda de la comunidad. La acción voluntaria y comunitaria es vista como una alternativa importante ante las dificultades del Estado de Bienestar y las insuficiencias de la institución familiar ${ }^{17}$. La barrera principal para la articulación de servicios de ayuda a la familia cuidadora es que su problemática no es visible social o políticamente. Las mujeres como principales artífices del cuidado a los ancianos precisan de una política que las libere temporalmente de los cuidados (centros de día, aestancias de día", servicios de compañía, etc.) para poder desarrollar su vida social. Las tensiones devienen más importantes en el caso de mujeres trabajadoras que tienen dificultades para compatibilizar su trabajo (o incluso vida familiar propia) con el cuidado. Es preciso potenciar sistemas de trabajo flexible o parcial que permitiese a la mujer cuidar a sus ancianos. De otro modo, la mujer se encuentra en una permanente posición de renuncia.

La familia ha dejado de prestar de forma exclusiva algunas de las funciones que la caracterizaban en las sociedades tradicionales: la unidad doméstica ha dejado de ser unidad de pro- 
ducción de objetos pero mantiene su función económica de producción de servicios y gestión de los recursos colectivos de capital y trabajo. Entre las funciones que continúa desempeñando se encuentran las asistenciales y de apoyo en relación a la salud. Respecto al cuidado de enfermos/as, la situación se repite. Preguntados los enfermos declaran que las familias han sido una fuente de apoyo fundamental. En el núcleo familiar se resuelve un elevado porcentaje de los episodios de enfermedad que sufren sus miembros. En los primeros síntomas de enfermedad la familia actúa como mediadora entre el enfermo y el sistema sanitario institucional. Según una encuesta del Cires sobre salud realizada en noviembre de 1990, un 43\% de los encuestados declaran haber comentado sus dolencias a un familiar en lugar de acudir directamente al médico (19\%). El cuidado al igual que la atención al enfermo se resuelve mayoritariamente en el ámbito informal. Según datos del CIS, la proporción de enfermos o discapacitados que son atendidos en la familia llega a alcanzar el $70 \%$. El peso que supone el cuidado de enfermos/as para la familia y más concretamente para la mujer se puede observar en la Tabla 4.

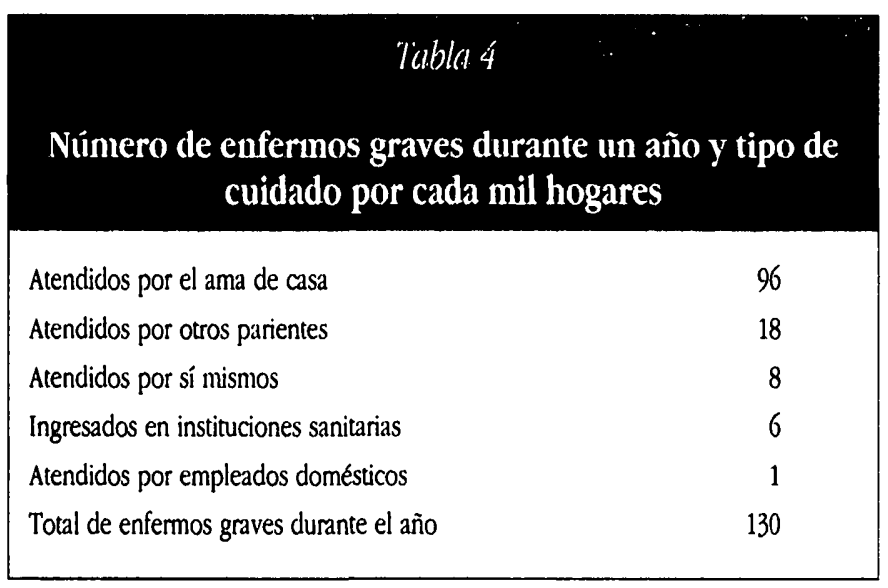

Fuente: Informe sobre -Desigualdad familiar y doméstica, CIS, Madrid 1985. Citado en el $V$ Informe Sociológico sobre la siluación social en España. Sociedad para todos en el año 2000. Fundación Foessa, p. 484.

Dentro de la organización doméstica es la mujer quien recibe la obligación estructuralmente adscrita de actuar como sujetos intermedios, entre el Estado y el resto de los ciudadanos en materia de salud. Se puede afirmar que tanto en el diseño como en la implementación de las políticas públicas se cuenta con el rol tradicional asumido por las mujeres para la lograr la consecución de las mismas. M. Ángeles DuRán afirma al respecto que: -las políticas de salud son programas colectivos de acción encaminados a convertir el sueño común de los ciudadanos (la salud para todos, èl pleno bienestar físico y mental de cada uno) en un objetivo político garantizado por el Estado. Pero tanto los valores y aspiraciones individuales como los objetivos y promesas garantizadas por la Constitución nacen y se asientan en una estructura social que pone el límite de sus recursos y de sus po- sibilidades. Por debajo de las redes formales de relación política se extiende una estructura más profunda, invisible habitualmente a los ojos del político y del legislador, que a fuerza de vivir inmersos en ella han llegado a olvidarla, si no es a preferir decididamente su ocultamiento" ${ }^{18}$. La mayor parte de los cuidados de la salud (los niños, los ancianos) tienen lugar en los extremos de ciclo de vida, que transcurre en el ámbito doméstico. Los incapacitados no llegan en su inmensa mayoría a abandonarlo nunca ${ }^{19}$.

A pesar de la relevancia del ámbito privado y cotidiano en el cuidado de la salud no existe una traducción en la esfera económica y política; de ahí su invisibilidad en los programas sanitarios. El Estado de Bienestar no asume la total responsabilidad sobre el enfermo ni cuando éste es institucionalizado. Cuando el enfermo es hospitalizado, se espera que la familia continúe cuidándolo. Además del afecto y la compañia, se espera de los/as acompanantes que asuman parte de los cuidados al enfermo relativos a su cuidado personal (alimentación, evacuación, limpieza, cambios de postura), vigilancia de instrumentos auxiliares (sonda, gotero) y aviso en caso de incidencias o deseos del enfermo. Forman parte de unas normas culturales muy arraigadas en la sociedad española las redes de solidaridad de la familia en caso de necesidad de alguno de sus miembros. La responsabilidad en las tareas de cuidado de enfermos continúa en manos femeninas ( $68 \%$ de los casos). Mientras que las mujeres se han adaptado al mercado laboral y han reajustado su dedicación en el ámbito doméstico, los varones permanecen en sus espacios tradicionales ${ }^{20}$. Las mujeres han protagonizado!

\begin{tabular}{|c|c|c|c|c|}
\hline \multicolumn{5}{|c|}{$\begin{array}{c}\text { Persona que se ocupa de atender a los miembros } \\
\text { de la fanilia enfermos seguin ocupación de las } \\
\text { entrevistadas (En porcentajes) }\end{array}$} \\
\hline \multirow{2}{*}{$\begin{array}{l}\text { Cuidar de los miembros } \\
\text { de la familia si } \\
\text { están enfermos }\end{array}$} & \multicolumn{4}{|c|}{ Ocupación de la mujer } \\
\hline & Total & Trabaja & Parada & $\begin{array}{l}\text { Se dedica } \\
\text { a labores } \\
\text { domésticas }\end{array}$ \\
\hline La mujer & $68 \%$ & $55 \%$ & $68 \%$ & $75 \%$ \\
\hline Ambos & 30 & 42 & 30 & 22 \\
\hline El varón & 1,4 & 2,3 & 1,7 & 0,9 \\
\hline Una tercera persona & 1,2 & 0,5 & - & 1,7 \\
\hline Total & $100 \%$ & & & \\
\hline Número de casos & $(695)$ & (213) & $(60)$ & $(422)$ \\
\hline
\end{tabular}

Fuente: Datos del Banco de Datos del CIS. Muestra nacional de 2.493 mujeres mayores de 18 años.

Estudio 2.194, octubre 1995. Elaboración propia. 
el tránsito del ámbito doméstico al ámbito laboral; en cambio, los varones al no incorporarse al ámbito doméstico incrementan las dificultades de esta transición. En ninguna de las situaciones ocupacionales de las mujeres se ha invertido su rol de cuidadoras. Sin embargo, entre las mujeres que trabajan, existe una tendencia a compartir las tareas del cuidado, a pesar de continuar siendo ellas las responsables en su mayoría tal y como se observa en la Tabla 5.
Las familias españolas cuidan, alimentan y atienden a ancianos, niños, parados ${ }^{21}$, minusválidos, enfermos de distinta gravedad, en muchos casos contando con servicios de apoyo muy escasos. La familia (si queremos ser precisas, la mujer) realiza una función de provisora de servicios sociales, que influye sobre la división de tareas en las familias y sobre las posibilidades de producción de nuevos roles familiares. Todo esto tiene la consecuencia de que el Estado se ve eximido, o al menos poco presionado, para solucionar activamente una larga lista de problemas sociales.
- Universidad de Barcelona.

1 Jane LEWTS Género, política familiar y trabajo remunerado y no remunerado. Duoda 13 (1997): 25-51.

' El trabajo no remunerado, como expresa DuRán (1994), se encuentra atrapado en un sistema de reglas propio, no escrito, no explicito, mal definido dentro del Derecho de la familia, no abiertamente pactado, que frecuentemente entra en contradicción con los principios generales del trabajo remunerado. Y puesto que gran parte de la cultura civica de la modernidad es heredera de las luchas y pactos sociales entre los asalariados y el capital. Los trabajadores (mejor utilizar slas trabajadoras.) no remunerados carecen de muchos de los derechos sociales y políticos de restantes trabajadores. En .The International Comparison of Gross Domestic Products: A Time and Gender Approach., Seminario celebrado en el Forum del European University Institute, Florencia.

3 Jane LEWIS remarca en este sentido que incluso entre los más influyentes intentos de establecer una tipología de los regímenes de bienestar, como es el caso de Gösta ESPING ANDERSEN, se ha ignorado la importancia del trabajo no remunerado y el papel importante que han cumplido primordialmente las mujeres en la realización del mismo en el ámbito familiar. El esquema de ESPING ANDERSEN partía de la relación entre trabajo y prestaciones sociales, donde trabajo se definía como trabajo remunerado y las prestaciones sociales como las políticas que permiten, fomentan o desincentivan la desmercantilización del trabajo. Ann OAKLEY (1986) pone el acento en el mismo aspecto señalando que la familia ha sido históricamente la principal proveedora de bienestar y que su importancia en este aspecto no muestra visos de menguar.

' Por eciudadanía civil, MARSHAll entiende los derechos de libertad de las personas, de expresión, de pensamiento y de confesión religiosa, derecho a poseer propiedad privada y a acordar contratos válidos y derecho de justicia. Por "ciudadanía política" se refiere al derecho a participar en el ejercicio del poder políico, al derecho a desempeñar un cargo público. La cciudadanía social. incluye los derechos relativos al bienestar económico y seguridad, el derecho a compartir la herencia social y vivir como un ser civilizado de acuerdo con los niveles predominantes de la sociedad. Es decir, se trata de garantizar ciertos derechos a beneficiarios materiales relacionados con el status de cciudadano. T. H. MarSHALL, Citizenship and Social Class (Cambridge: Cambridge University Press, 1950).

"Es posible afirmar que las diferencias se han reducido apreciablemente en los últimos años en el ámbito del trabajo remunerado, con la incorporación de un mayor número de mujeres al mercado laboral (aunque no necesariamente ha ocurrido lo mismo en el caso de la remuneración, la categoría del puesto de trabajo y la duración de la jomada laboral todos los datos indican que el reparto de trabajo no remunerado se ha modificado muy poco. En un reciente artículo aparecido en El País (15-31999) se afirma en base a un estudio realizado por el sociólogo Gerardo MeIL, que Sólo en ocho de cada cien familias españolas se produce un reparto de las tareas domésticas. Según MeIl, ésa es la causa del rápido crecimiento de las tasas de divorcio de la última década (del 1,5 al 1,9 por 1.000); aunque tampoco se está produciendo una desestabilización grande en la familia española, es más cada vez tiene mayor importancia: Sirve de hospital, de centro de acogida de drogas, de geriátrico y de colchón para el paro.

" En Fundación Foessa, ed., Quinto informe sociológico sobre la situación social en España: Sociedad para todos en el año 2000 (Madrid: Fundación Foessa, 1994), p. 481.
' Según la argumentación de Víctor PÉREz Díaz Si la familia espanoola ha continuado manteniendo un elevado protagonismo en el sistema de bienestar español, ello se debe en gran medida a que la responsabilidad que siente por la felicidad de sus miembros se funda en unos afectos cuya solidez no depende de circunstancias variabales, tales como la mayor o menor provisión pública de prestaciones sociales. sino que obedece al objetivo primordial de preservar la cohesión de sus componentes por encima de dificultades y obstáculos de diverso tipo. Todo ello revierte en el afianzamiento de los lazos y la unidad familiar puesto que -protegiendo a sus miembros, las familias se autoalimentan, se aseguran su propia supervivencia. En .La familia en el sistema de bienestar español. Una reflexión general y una discusión empírica. Papeles de economia española 77 (1998), p. 29.

${ }^{8}$ Es interesante la aportación al análisis sociológico de las ideologías relacionadas con la profesión médica que Jesús M. de Miguel realiza en La amorosa dictadura (Barcelona: Anagrama, 1984), 235 pp.

$?$ Mary NASH, Historia e historiografia de las mujeres españolas. en M. Antonia GARCíA DE LEÓN et al., Sociologia de las mujeres españolas (Madrid: Editorial Complutense, 1996), p. 397.

li" Jesús M. de Miguel, Estructura y cambio social en España (Madrid: Alianza Editorial, 1998), p. 452.

1 Enrique Gil Calvo, Edad y género• en María Antonia García de León (coord.), Sociologia de las mujeres españolas (Madrid: Editorial Complutense, 1996). p. 21.

1. Recientemente (El País 10 de febrero, 1999) se han presentado tres proposiciones de ley en el Congreso relacionadas con los permisos de maternidad. Según estas proposiciones de ley, las familias que adoptan o acojan a menores de cualquier edad tendrán períodos de permiso por maternidad, al igual que las familias biológicas. Estas medidas pretenden la conciliación de la vida familiar y laboral.

1. Hasta 1994 la situación derivada de la matemidad de la mujer trabajadora se equiparaba a la incapacidad laboral por enfermedad común, por lo que su tratamiento normativo a efectos de protección social era semejante al de esta contingencia. En consonancia con este cambio de concepto, en diciembre de 1994 la cuantía de la prestación económica se eleva desde el $75 \%$ del salario al $100 \%$. La duración del descanso es de 16 semanas ininterrumpidas ampliables a 18 en caso de parto múltiple. El padre puede disfrutar altemativamente de cuatro de las últimas semanas del permiso, aunque no existen datos que permitan conocer cuántos hombres han decidido acogerse a este derecho en vez de sus esposas trabajadoras.

1. A este respecto, el papel de las abuelas a cargo de quienes muchas veces quedan los nietos/as merece ser estudiado en profundidad. El incremento de la esperanza de vida y la mejora de la salud y la calidad de vida de las personas ancianas permite a muchas mujeres adultas pedir a sus madres prestaciones que antes no estaban en condiciones de ofrecer.

15 Según las cifras, en 1980 el grupo de edad de más de sesenta y cinco años sólo representaba en España el $11 \%$ de la población, pero al inicio de la década de 1990 ya ha pasado a ser el 15\%. Para el año 2050 se prevé que España tenga más de nueve millones de personas de más de 65 años. El impacto social de este fenómeno está iniciándose, pero sus efectos obligan a plantear cambios acerca del modelo de sociedad, de las necesidades sociales y sanitarias, así como de las características y orientación de las políticas sociales y de bienestar. 
1. Como señala el estudio realizado por Josep Rodríguez, el elemento determinante ''n el proceso de cuidar es el momento en que el anciano se queda solo (pierde a su cónyuge) y progresivamente necesita a alguien que le atienda y cuide. El $76 \%$ de los ancianos conviven con sus familiares porque la familia no quiere dejarlos solos (40\%) o porque considera que necesita a alguien que los cuide (36\%). En Envejecimiento y familia (Madrid: Centro de Investigaciones Sociológicas 137, 1994), p. 73.

"No existe todavia ninguna política que vaya dirigida directamente a ayudar a la familia en sus necesidades o problemas cuidando ancianos. El Plan Gerontológico Nacional plantea el desarrollo de acciones que directa o indirectamente pueden suponer una ayuda a la familia. Son políticas cuyo objetivo es incentivar cultural, social y fiscalmente a la familia para que asuma el papel cuidador. El Pla Integral de la Vellesa de Catalunya propone medidas en el mismo sentido. En esta concepción la familia es vista como mecanismo para reducir la presión asistencial de este grupo de población.

Ik La mediación invisible: De las utopías sociales a los programas políticos en materia de salud• en Jesús M. de MiGuel, Javier YUSTE y María Ángeles DuRáiv, El futuro de la salud (Madrid: Centro de Estudios Constitucionales, 1988), p. 121.
1) La Encuesta del Centro de Investigaciones Sociológicas de 1984 sobre ‘Desigualdad familiar y doméstica. estimaba que una de cada diez amas de casa tenían un incapacitado entre sus parientes próximos. Se estimaba que sólo un $10 \%$ de los incapacitados recibían de una institución la mayor parte del tiempo de cuidado requerido.

3i. La Seguridad Social contempla en sus presupuestos una partida para subvencionar a los acompañantes de los enfermos desplazados fuera de su lugar de residencia, aunque se dirijan más a subvencionar los gastos originados por el desplazamiento que el cuidado prestado por el acompañante.

"Según Ana M. Gunlév .El mercado de trabajo funciona (no estalla) y las altísimas tasas de paro se soportan en España gracias a que las familias comparten sus ingresos (procedentes de empleos seguros, precarios o de la economía sumergida o de las prestaciones sociales), acogiendo a parados y necesitados en general. (Bellaterra: $\mathrm{Pa}$ pers 53,1997$)$ p. 56.

\section{Bibliografia}

Dominguez AlCon, Carmen, y M. Teresa Bazo, -Los cuidados familiares de salud en las personas ancianas y las políticas familiares. (Madrid: Revista Española de Investigaciones Sociológicas 73, 1996), pp. 45-56.

Domínguez Alcón, Carmen, Envejecimiento y familia. (Bellaterra: Papers 40, 1993), pp. $75-88$.

Domínguez Alcon, Carmen, Familia, cuidado informal y políticas de vejez en SIMON, y Lincla CROMPTON HODGES Enfermería gerontológica. Adaptación al proceso de envejecimiento (Méjico: McGmwHill, 1997), pp. 464-476.

Durín, M. Angeles, El trabajo de la mujer en España: Un estudio sociológico (Madrid Técnos, 1972).

DuRín, M. Angeles, La jomada interminable (Barcelona: Icaria, 1987).

ESPING-ANDERSEN, Gösta, Los tres mundos del Estado del Bienestar (Valencia: Alfons el Magnànim, 1993)

Fundación Foessa, Quinto informe sociológico sobre la situación de España 19831993: La sociedad para todos en el año 2000 (Madrid: Fundación Foessa, 1994), 2 vols., $2313 \mathrm{pp}$.

GARCIA DE LEÓN, M. Antonia et al., Sociología de las mujeres españolas (Madrid: Editorial Complutense, 1990), $421 \mathrm{pp}$

GarRIDO, Luis y, Enrique Gil Calvo, Estrategias familiares (Madrid: Alianza, 1993).

Gil. Calvo, Enrique, Participación laboral de la mujer, natalidad y tamaño de cohortes, Revista Española de Investigaciones Sociológicas 47 (1989): 137-175.

Guilún, Ana M., ·Regímenes de bienestar y roles familiares: un análisis del caso espanol. (Bellaterra, Papers 53, 1997), pp. $45-63$

HaRDING, Sandra (ed.) Feminism and Methodology (Indiana: Indiana University Press, 1987), $193 \mathrm{pp}$.

IGLESIAS DE UsSeL, Julio, y Lluís FLAQUER, ·Familia y análisis sociológico: El caso de España. Revista Española de Investigaciones Saciológicas 61 (1993): 57-75.

Instituto de la Mujer, Cronos Dividido: Uso del tiempo y desigualdad entre mujeres y - bombres en España, Serie Estudios, $n^{2} 26$ (Madrid: Ministerio de Asuntos Sociales, 1990), $261 \mathrm{pp}$.

Instituto de la Mujer, Expectativas femeninas sobre redes de cuidados de bijos, (Madrid; Ministerio de Asuntos Sociales, 1990), 121 pp.
Instituto de la Mujer, El trabajo doméstico y la reproducción social (Madrid: Ministerio de Asuntos Sociales, 1991).

Instituto de la Mujer, Mujeres, trabajos y politicas sociales: Una aproximación al caso expañol (Madrid: Ministerio de Asuntos Sociales, 1997), 240 pp.

IzQuerdo, M. Jesús, El malestar en la desigualdad (Madrid: Cátedra, 1998), 409 pp.

LEWIS, Jane .Género, política familiar y trabajo remunerado y no remunerado. Duoda 13 (1997): 25-51.

MAck, Dana, The Assault on Parenthood: How Our Culture Undemines the Family (Simon and Schuster, 1997), $368 \mathrm{pp}$

MAR-KLOSE, Marga y Anna NOS COLOM, Itinerarios de vida: Estrategias de educación, trabajo, nupcialidad y fecundidad. (Madrid: Centro de Investigaciones Sociológicas, 1999). Publicado en la Colección .Opiniones y actitudes. En prensa.

MIGuEl, Jesús M. de, YUSTE, Javier y DuRÁv, María Ángeles, El futuro de la salud (Madrid: Centro de Estudios Constitucionales, 1988).

Miguel, Jesús M. de, Estructura y cambio social en España (Madrid: Alianza Editorial, 1998), $681 \mathrm{pp}$

PÉREZ DiAZ, Victor $e t$ al. sLa familia en el sistema de bienestar español. Una reflexión general y un discusión empírica. Papeles de economia española 77 (1998): 24-39.

PÉrez-Diaz, Victor, Elisa Chulá y Berta Álvarez-Miranda, Familia y sistema de bienestar: la experiencia española con el paro, las pensiones, la sanidad y la educación. (Madrid: Fundación Argentaria, 1998), 184 pp.

ROdRIGUEZ, Josep A., Envejecimiento y familia (Madrid: Centro de Investigaciones Sociológicas 137, 1994), $115 \mathrm{pp}$.

Russell HoCHSCHIEl.D, Arlie, The Second Shift (Nueva York: Penguin Books, 1989).

RuSSELL HOCHSCHIEL, Arlie, The Time Bind: When Work Becomes Home and Home Becomes Work (Nueva York: Metropolitan, 1997), $316 \mathrm{pp}$.

SENSAT, Nuria y VARELA, R., LLa políticas dirigidas a las mujeres: La acción pública para la igualdad entre los sexos" en Ricard Gomà y Joan SuBIRATS (coords.), Politicas puiblicas en España: Contenidos, redes de actores y niveles de gobierno (Barcelona: Ariel, 1998), pp. 341-361. 


\section{Cuadernos de DERECHO PUBLICO

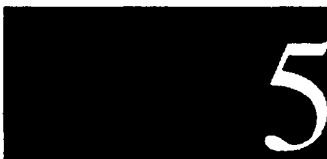 \\ SEPTIEMBRE-DICIEMBRE 1998 \\ EL PRINCIPIO \\ DE PROPORCIONALIDAD \\ INAP}

\section{Instituto Nacional de Administración Pública}

Nueva publicación editada por el Instituto Nacional de Administración Pública, los Cuadernos de Derecho Público, dedicada a los estudios y análisis relativos a la organización y funcionamiento del Estado desde una perspectiva global, interdisciplinaria, que aspira como tal publicación, integradora y rigurosa en sus planteamientos, a abrirse un espacio en el marco de la publicística actual.

\section{CONSEJO DE REDACCIÓN}

Presidente: José Luis Meilán Gil. Vocales: Enrique Arnaldo Alcubilla, José Luis Cascajo Castro, Francesc de Carreras Serra, Ignacio Díez-Picazo Giménez, Germán Fernández Farreres, Rafael Femández Montalvo, Carmen González Fernández, Alejandro Menéndez Moreno, Juan Antonio Ortega Díaz-Ambrona, José Luis Piñar Mañas, Juan Antonio Puigserver Martínez, María Rosa Ripollés Serrano, Luis Ignacio Sánchez Rodríguez, José Antonio Souto, Carles Viver i Pi-Sunyer, Tomás S. Vives Antón.

\section{Director}

Luis Aguiar de Luque

\section{Secretario}

Ricardo Blanco Canales

\section{Coordinación}

Centro de Publicaciones del INAP

Edita:

MINISTERIO DE ADMINISTRACIONES PÚBLICAS Instituto Nacional de Administración Pública

\section{Suscripciones y Administración:}

Instituto Nacional de Administración Pública Publicaciones Periódicas Atocha, 106. 28012 Madrid Teléfono: 3493235

\section{Precios:}

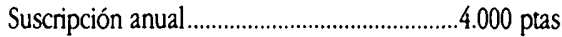

